\title{
A comparison study on integrating electronic health records into priority simulation in undergraduate nursing education
}

\author{
Weihua Zhang, Darla Ura, Barbara Kaplan \\ Nell Hodgson Woodruff School of Nursing, Emory University, Atlanta, Georgia, USA.
}

Correspondence: Weihua Zhang. Address: Nell Hodgson Woodruff School of Nursing, Emory University, Atlanta, Georgia, USA. Email: wzhang3@emory.edu

Received: March 11, 2014

DOI : 10.5430/jnep.v4n7p123
Accepted: April 8, $2014 \quad$ Online Published: May 8, 2014

URL: http://dx.doi.org/10.5430/jnep.v4n7p123

\section{Abstract}

Background: Electronic Health Records (EHR) make real time point of care efficient. We implemented EHR into our existing simulation exercise and attempted to evaluate the students' perception on the effectiveness of the simulation in our undergraduate nursing students in comparison to the same cohort who had the same simulation without using electronic health records in the prior year.

Purpose: The main purpose of this study is to assess the difference on simulation effectiveness perceived by the students in the group with and without utilizing EHR in simulation exercise.

Method: A descriptive research design and convenience sampling was used to compare the effectiveness of perception data collected from the students after the simulation in these two groups. The difference in perception was compared by using the $t$-test.

Result: There is no statistically significant change in students' perception $(t=.79, p=.42)$ between the simulation (SIM) and the simulation with EHR integration (SIMEHR) group. The confidence in providing care and knowing the patient by utilizing electronic health records has been reported as between somewhat agree to strongly agree.

Discussion: Integrating EHR into simulation did not significantly change students' perception on simulation effectiveness. The implication from this study is that the integration of EHR into the simulation can be accomplished with careful prior planning with emphasis on introducing the strategies that enhance students' ability to get familiarized with the EHR system.

\section{Key words}

Electronic health records, Simulation, Undergraduate nursing education, Priority simulation

\section{I ntroduction}

Currently, health care settings in the United States are becoming more electronically controlled. Federal mandates regarding the adoption of the Electronic Health Record (EHR) in health care settings has in turn caused the incorporation of the use of EHR to support clinical decision making in nursing education of paramount importance. The main purpose of adding the utilization of electronic health records in simulation in nursing education is to meet the new demands of health 
care for the U.S. population in the 21st century and to fulfill the requirements as set forth through the implementation of the Affordable Care Act. Prioritizing nursing management is critical in nursing practice and is a particular challenge to effectively teach to nursing students. With each nurse having more than one patient at any given time, prioritizing the care can be pivotal to patient outcome. We have been conducting a nursing prioritizing simulation over the last few years without accessing electronic health records. This critical decision on prioritizing is based on available information that helps nurses to know the patient and determining what needs to be done first ${ }^{[1]}$. With the Federal mandates and electronic health records now readily available in hospital settings, we recognized the need to train nursing students to be comfortable navigating and obtaining pertinent information prior to graduation. Training nurses to be confident in navigating EHR systems and providing quality and safe care while prioritizing nursing care is one of the essentials of nursing education proposed by the American Association of Colleges of Nursing ${ }^{[2]}$. Therefore, we added the electronic health records access prior to and during our existing nursing prioritizing simulation. The purpose of this manuscript is to evaluate nursing students' perception of simulation effectiveness comparing with the prior simulation group using traditional hard chart to access patient's information to the current group using the electronic chart to access patient's information. The intention of reporting the procedures and findings is specific to the university and is not intended to be generalized beyond this institution.

\section{Literature review}

\subsection{Electronic Health Records}

The emphasis on using EHR has been intensified as it is now a federal mandate in the U.S. ${ }^{[3]}$. According to this mandate, all health care facilities must replace traditional paper charting with EHR in order to receive full reimbursement for the services provided by the year $2015^{[4,5]}$. The Office of the National Coordinator (ONC) for Health Information Technology reported that more and more providers and hospitals nationwide understand why electronic health record (EHR) adoption and achieving meaningful use is critical to improving patient care. Since the EHR Incentive Programs began in January, $2011^{[6]}$, 54 percent of the Medicare- and Medicaid-eligible professionals had registered for the meaningful use incentive program as of August $2012{ }^{[7]}$. Due to this mandate, it is anticipated that most nursing students will step into the clinical practices where EHRs are utilized.

\subsection{Utilizing EHR in nursing education and QSEN}

To ensure the students are prepared to enter this technology rich working environment, our school has adopted the use of an EHR into the undergraduate nursing program. It is essential that the curriculum meet nursing accreditation regarding the adoption of informatics as well as QSEN (Quality and Safety Education for Nurses) competency standards regarding the delivery of safe, efficient, and quality care ${ }^{[8,9]}$. Training nurses to be confident in navigating EHR system and provide quality and safe care at the point of care is one of the essentials in nursing education proposed by the American Association of Colleges of Nursing ${ }^{[2]}$. The objective of point of care (POC) is for nurses to retrieve patient data and document at the bedside. Research has shown that POC documentation is more accurate and enhances continuity and safety for the patient ${ }^{[10-12]}$. In our new pedagogy, students will learn in the context of nursing processes in a simulated (SIM) environment using the electronic health records (EHR) to provide quality and safety patient care in a real-world setting (SIMEHR). In addition learning how to document in the EHR, students will learn essential skills for discerning data, the value of accurate documentation and how their interaction with the EHR impacts the care team as a whole.

\section{Method}

\subsection{Sample}

A descriptive research study design and convenience sampling were used to compare students on effectiveness perception. The study spanned a 2-year time period with all senior nursing students in the final semester of the nursing program. The 
prior year students in the simulation group (SIM) used a teacher made paper chart at the patient's bedside $(\mathrm{n}=99)$ and the following year senior students were in the simulation with EHR access (SIMEHR) group $(\mathrm{n}=110)$.

\subsection{I nstrument}

METI (Medical Education Technologies Inc) simulation effectiveness tool was used for the students post simulation. METI contains a 13-item 3-point ordinal scale instrument. Two subscales, "confidence" and "learning” were noted with Cronbach's alpha of .88 (confidence) and .87 (learning) has been reported by the author ${ }^{[13]}$. The same instrument was used for both years with an exception that two extra questions related to evaluation on integration of EHR to current simulation for the SIMEHR group were added.

\subsection{Procedure}

Students participated in the nursing prioritizing simulation in pairs. The assessment component includes but is not limited to listening to nurse's change of shift report, reviewing the patient's chart (either a teacher made paper chart at the patient's bedside - previous group versus the EHR - current group), and bedside physical assessment of the patients. Students are then required to care for the three simulated patients. The simulation was designed for students to assess the condition of three simulated patients and to come up with the decisions on delegating, implementing, and continuously evaluating the appropriateness of the care provided to the patients. The three patients are: (1) abdominal aortic aneurysm (AAA) with impending rupture, (2) lower gastrointestinal bleeding with sudden active bleeding, and (3) a diabetic patient experiencing hypoglycemia. The simulation allows two students to practice prioritizing and delegating skills when planning for anticipated patient needs as well as when dealing with unexpected events. During the exercise, both AAA and GI bleeder developed hypotension, tachycardia, and tachypnea. Other accompanied symptoms including severe and persistent abdominal pain for the AAA patient and severe dizziness for the GI bleeding patient. The third patient received insulin injection due to her elevated morning glucose level. However, she was not able to eat and developed symptoms associated with hypoglycemic shock. The simulation provided students some opportunities to practice their critical thinking skills by identification of knowledge gaps, setting priorities, accurately assessing needs, re-evaluating plans and providing interventions as needed. They are expected to correctly identify nursing actions and modify the nursing plan as the patient condition changes. In addition to the above mentioned exercise, the SIMEHR group is required to utilize electronic health records to gather necessary information and thoughtfully analyze findings to plan care strategically. This simulation was the first time that students used the newly purchased EHR system. The students were required to review a "scavenger hunt”- a game designed to help students to find contents such as "x-ray report”, "complete blood counts”, "glucose level”, "vital signs", and others, which is prepared by the manufacturer prior to their simulation time in order to familiarize them with the system components. The link for the scavenger hunt was posted on the Blackboard course website. Other strategies are integral to the success of implementing the EHR into simulation program was for it to be "live" and to reflect the actual date and time that the students would participate in the simulation. Therefore, faculty worked with the EHR liaison from the vendor to ensure each student group would have accurate access to the scheduled time for participation in the "real-time" simulation.

In addition, these students could access the EHR of the three designated patients prior to the simulation as well as during the simulation allowing them to analyze medications and lab results in real time. Debriefing was conducted after the simulation led by the faculty member who conducted and observed the simulation. At the end of the simulation, students were asked to complete a simulation effectiveness perception survey.

\section{Results}

\subsection{Demographic characteristics}

The demographic characteristics are comparable between the two groups (see Table 1). The mean age for the SIM group was 27.6 years old and the mean age for the SIMEHR group was 26.3. Most students are female in both groups. There 
were 55.6\% Caucasians, 18.2\% Black, 5\% Hispanic and 8.1\% Asian in SIM group and 45.5\% Caucasians, 21.8\% Black, 2.7\% Hispanic, and 9.1\% Asian in the SIMEHR group.

Table 1. Demographic Characteristic of 2011 and 2012 Group

\begin{tabular}{lllll}
\hline Students & SIM Group (N = 99) & & \multicolumn{2}{l}{ SIMEHR Group (N = 110) } \\
\hline \multirow{2}{*}{ Age } & Range & Mean (SD) & Range & Mean (SD) \\
& $22-54$ & $27.6(6.8)$ & $21-45$ & 26.3 (5.3) \\
\hline \multicolumn{1}{c}{ Gender } & $\mathbf{N}$ & $\mathbf{\%}$ & $\mathbf{N}$ & $\mathbf{\%}$ \\
$\quad$ Male & & & & 13.6 \\
$\quad$ Female & 6 & 6.0 & 15 & 96.4 \\
Race & 93 & 93.9 & 95 & \\
$\quad$ White & & & & 45.5 \\
$\quad$ Black & 55 & 55.6 & 50 & 21.8 \\
$\quad$ Hispanic & 18 & 18.2 & 24 & 2.7 \\
Asian & 5 & 5.1 & 3 & 9.1 \\
$\quad$ Others & 8 & 8.1 & 10 & 21 \\
\hline
\end{tabular}

\subsection{Data analysis}

The evaluation of effectiveness was based on students' responses to the learning experience by using a short METI survey online by the students attended the simulation at our school. Survey results were tabulated to validate the value of utilizing an SIMEHR within the curriculum at our institution. Both group's effectiveness perception survey were examined for outliers and assessed for the assumption of normality using skew and kurtosis. The two sets of data have normal distribution. All statistical analyses were performed using the Statistical Package for Social Sciences (SPSS) software.

We used the total score of the first 13 questions (same questions used for both years) and found no significant difference ( $t$ $=.79, p=.42$ ) between the SIM and the SIMEHR group (see Table 2). The total score of the first 13 questions was 34.07 in 2013 class and 33.42 in 2012 class with 106 out of 110 responded to the survey in 2013 vs. only 36 out of 99 students responded to the survey in 2012. Fewer participants for the 2012 survey could be the result of 1 ) the participation of online survey was not mandatory and 2) missing date from the blackboard if it is not downloaded the same year. We accessed 2012 survey on the blackboard in year 2013.

We added two items related to using electronic health records in addition to METI to the 2013 class. The first question was: "My ability of providing care to my patients was enhanced by obtaining information retrieved from electronic health records;" and the second question was: "My confidence in providing care to my patients was increased by utilizing electronic health records." The average score on these two questions were 2.22 and 2.18 (with " 0 " don't agree and "3" strongly agree with the statement).

Table 2. Comparison of SIM group with the SIMEHR group on perceptions of effectiveness of simulation

\begin{tabular}{llllll}
\hline & \multicolumn{2}{l}{ Differences } & \multicolumn{2}{c}{ 95\% CI of the difference } \\
\cline { 2 - 6 } & $\boldsymbol{t}$ & Df & $\boldsymbol{p}$ & Lower & Upper \\
\hline SIM and SIMEHR & 0.797 & 140 & .427 & .96 & 2.25 \\
\hline
\end{tabular}

\section{Discussion/ I mplications for practice}

Simulations have been widely used in undergraduate nursing education around the world ${ }^{[14]}$. Simulations can reduce students' anxiety level and increase their caring ability in crisis situation ${ }^{[15]}$. Simulations also have been shown to increase students' confidence in patient care ${ }^{[16]}$. To date, there are no reported studies on incorporating electronic health records 
into simulations in nursing education. The results of this study indicated that there were no significant changes in students' perception over the effectiveness of the simulation between the group using the paper charting and the group using the electronic medical record. We interpreted these findings positively as the inference is that students did not view the EHR as an impediment for their learning process. This could suggest that while designing simulations in the future we could integrate the EHR with a reasonable expectation of seamless transition for our students. In addition, with neutral student perceptional changes in integration of EHR into the simulation suggests that the transition from utilizing paper chart to electronic chart should be started from early nursing education. From our experience with integrating EHR to the pre-existing simulation, we were able to implement it into our current curriculum successfully with aforementioned proper planning. Other possible reason could be that our students are technologically savvy and made adaptation to the new technology rather smoothly. Positive student comments on their ability and confidence in using the EHR and providing patient care further support this finding.

This study was limited by the instrument selected as it lacked questions regarding the impact of the paper chart in student's confidence or ability to care for the simulated patients in the previous year of the study. Paper charting has been a historic issue with safe and quality health care delivery. In our simulation, we eliminated the possible confusions related to misinterpretation of the handwritten physician's orders, the Medical Administration Record (MAR) and the general diagnosis and plan of care. The PRN order as hand-written on the MAR was frequently overlooked and necessitated the student's making an SBAR (situation, background, assessment and recommendation) call to the "provider" when it was not warranted in our previous simulation while electronic health records were not utilized. The SIMEHR group had no problem in reading or interpreting the chart which could also contribute their increased self-confidence. Future studies shall explore the electronic health records in relation to their self-confidence, decision making ability comparing with those who use the paper chart.

Due to the seamless transition in use of the EHR and the positive research findings, the EHR and simulation integration will be sustained in our undergraduate nursing curriculum. We plan to continue to implement the use of computer technology in existing simulation exercises and to utilize the SIMEHR as a tool to reinforce basic nursing principals and competencies needed for successful passing of NCLEX - RN (National Council Licensure Examination). Electronic health records will enhance patient safety and quality care by making interdisciplinary team members' plan of care transparent to the entire team members. Less iatrogenic errors can be predicted by misinterpretations due to illegible handwritten notes and orders. Patient centered care can be achieved by the care team with high confidence in decision making. Hospital could adopt this pedagogy in training the newly graduated nurses to increase their confidence in delivering effective care to their patients.

\section{References}

[1] Kaplan B, Ura D. Use of multiple patient simulators to enhance prioritizing and delegating skills for senior nursing students. Journal of Nursing Education. 2010; 49(7): 371. PMid:20411859 http://dx.doi.org/10.3928/01484834-20100331-07

[2] Mailloux CG. Using The Essentials of Baccalaureate Education for Professional Nursing Practice (2008) as a framework for curriculum revision. Journal Professional Nurse. 2011; 27(6): 385-9. PMid:22142915 http://dx.doi.org/10.1016/j.profnurs.2011.04.009

[3] Blumenthal D, Tavenner M. The "meaningful use" regulation for electronic health records. The New England journal of medicine. 2010; 363(6): 501-4. PMid:20647183 http://dx.doi.org/10.1056/NEJMp1006114

[4] Mgma Government Affairs Department. The Federal EHR incentive program: achieving 'meaningful use'. MGMA connexion / Medical group Management Association. 2010; 10(8): 14-6.

[5] Robeznieks A, Lubell J. 'Meaningful use' at last. Regs aim to boost quality, safety and efficiency. Modern healthcare. 2010; 40(1): 4. PMid:20088268

[6] Centers for Medicare \& Medicaid Services. CMS Medicare and Medicaid EHR Incentive Programs: Milestone Timeline. 2012.

[7] HealthIT.gov. Benefits of Electronic Health Records (EHRs). 2014. 
[8] Disch J. QSEN? What's QSEN? Nursing outlook. 2012; 60(2): 58-9. PMid:22306706 http://dx.doi.org/10.1016/j.outlook.2012.01.001

[9] Dycus P, McKeon L. Using QSEN to measure quality and safety knowledge, skills, and attitudes of experienced pediatric oncology nurses: an international study. Quality management in health care. 2009; 18(3): 202-8. PMid:19609190 http://dx.doi.org/10.1097/QMH.0b013e3181aea256

[10] Ehrmeyer SS. Plan for quality to improve patient safety at the point of care. Annals of Saudi medicine. 2011; 31(4): 342-6. PMid:21808107 http://dx.doi.org/10.4103/0256-4947.83203

[11] Briggs B. Patient safety driving point-of-care I.T. plans. Health data management. 2004; 12(10): 56, 8, 60.

[12] Hagland M. Point-of-care protection. Hospitals move to enhance patient safety in common but high-vulnerability situations. Healthcare informatics: the business magazine for information and communication systems. 2004; 21(6): 80.

[13] Elfrink VL, Leighton K, Ryan-Wenger N, Doyle TJ, Ravert P. History and Development of the Simulation Effectiveness Tool. Clinical Simulation in Nursing. 2012; 8(6): 199. http://dx.doi.org/10.1016/j.ecns.2011.12.001

[14] Dunnington RM. The nature of reality represented in high fidelity human patient simulation: philosophical perspectives and implications for nursing education. Nursing philosophy: an international journal for healthcare professionals. 2014; 15(1): 14-22. PMid:24320978 http://dx.doi.org/10.1111/nup.12034

[15] Khalaila R. Simulation in nursing education: an evaluation of students' outcomes at their first clinical practice combined with simulations. Nurse education today. 2014; 34(2): 252-8. PMid:24060462 http://dx.doi.org/10.1016/j.nedt.2013.08.015

[16] Abe Y, Kawahara C, Yamashina A, Tsuboi R. Repeated scenario simulation to improve competency in critical care: a new approach for nursing education. American journal of critical care: an official publication, American Association of Critical-Care Nurses. 2013; 22(1): 33-40. PMid:23283086 http://dx.doi.org/10.4037/ajcc2013229 